OPEN ACCESS

Edited by: Martin Lepage,

McGill University, Canada

Reviewed by:

Kiyotaka Nemoto,

University of Tsukuba, Japan

Pablo Cuesta

Universidad de La Laguna, Spain

${ }^{*}$ Correspondence:

Guogiang Xing

gxing99@yahoo.com

Ying Han

hanying@xwh.ccmu.edu.cn

Specialty section:

This article was submitted to

Neuroimaging and Stimulation,

a section of the journal

Frontiers in Psychiatry

Received: 04 June 2018 Accepted: 21 November 2018 Published: 06 December 2018

Citation:

Lin L, Xing G and Han Y (2018)

Advances in Resting State

Neuroimaging of Mild Cognitive

Impairment. Front. Psychiatry 9:671.

doi: 10.3389/fpsyt.2018.00671

\section{Advances in Resting State Neuroimaging of Mild Cognitive Impairment}

\author{
Li Lin ${ }^{1}$, Guoqiang Xing ${ }^{2 *}$ and Ying Han ${ }^{1,3,4,5 *}$ \\ ${ }^{1}$ Department of Neurology, XuanWu Hospital of Capital Medical University, Beijing, China, ${ }^{2}$ Department of Imaging \& Imaging \\ Institute of Rehabilitation and Development of Brain Function, The Second Clinical Institute of North Sichuan Medical College, \\ Nanchong Central Hospital, Nanchong, China, ${ }^{3}$ Beijing Institute of Geriatrics, Beijing, China, ${ }^{4}$ National Clinical Research \\ Center for Geriatric Disorders, Beijing, China, ${ }^{5}$ Center of Alzheimer's Disease, Beijing Institute for Brain Disorders, Beijing, \\ China
}

The rapidly increasing number of patients with Alzheimer's disease (AD) worldwide has become a major public concern. Mild cognitive impairment (MCl), characterized with accelerated memory decline than normal aging, is a stage between cognitively unimpaired and dementia. Identification of $\mathrm{MCl}$ in the Alzheimer's continuum from normal aging, is important for early diagnosis and improved intervention of AD. The imaging technique has been extensively used for diagnose and understanding the mechanisms of $\mathrm{MCl}$. Firstly, we review the recent progresses in the research framework of $\mathrm{MCl}$ depending on the clinical and/or biomarker findings. Secondly, we cover studies that use of rs-fMRI (resting state functional magnetic resonance imaging) for the brain activities and functional connectivity between normal aging and $\mathrm{MCl}$. Other methodologies and multi-modal studies for investigating the mechanism and early diagnosis of $\mathrm{MCl}$ are also discussed. Finally, we discuss how genetic and environmental factors such as education could interact with in $\mathrm{MCl}$. Overall, $\mathrm{MCl}$ is a heterogeneous state and employing resting state neuroimaging with other AD biomarker approaches will be able to target in the more precise population and AD-related pathology process.

Keywords: fMRI, resting state, mild cognitive impairment, Alzheimer's disease, functional connectivity

\section{INTRODUCTION}

Alzheimer's disease (AD) is the most common cause of dementia in older adults worldwide. There will be one new case of dementia every $3 \mathrm{~s}$. By 2030, the number of people with the disease is expected to rise to more than 70 million (1). The burden of AD patients on themselves, their families, and society has risen considerably and attracted significant attention. Although much attention has been paid to $\mathrm{AD}$, no effective treatment for $\mathrm{AD}$ are available to date. Nowadays, drugs may produce some short-term improvement in cognitive function but they cannot slow down or stop the progression of the pathological damages if one is currently diagnosed $\mathrm{AD}$ dementia which is in the late stage of the disease. Despite of this, diagnosis at the early phase of the disease, like preclinical stage, or prodromal stage, may represent a good opportunity for interventions (2).

To better diagnose and define clinical stages of $\mathrm{AD}$, two major diagnostic criteria, the National Institute of Aging and Alzheimer's Association (NIA-AA) criteria and the International Working Group(IWG) criteria, for AD were proposed (3-5). The NIA-AA criteria describes an entire clinical spectrum and the related biomarkers of $\operatorname{AD}(4,5)$. Mild cognitive impairment $(\mathrm{MCI})$ is 
an intermediate stage between cognitively unimpaired and dementia. Fifteen to twenty percentage of people aged 65 or older have MCI and these patients have a high probability of converting to $\mathrm{AD}$, at an average of $32 \%$ in 5 years (6-8). Identifying these individuals with MCI could be an effective strategy for early diagnosis and treatments to delay and/or halt $\mathrm{AD}$ progression toward an irreversible full brain damage (9).

Toward greater understanding of the mechanisms of $\mathrm{AD}$, not only clinical or cognitive tests, but also biological methods have to been used. Neuroimaging studies have recently begun to illuminate structural and functional brain abnormality in early $\mathrm{AD}$ and even constitute a vital part of the research framework about $\mathrm{AD}$ (5). Resting-state functional magnetic resonance imaging (rs-fMRI), which measures the spontaneous fluctuations of blood oxygenation level-dependent (BOLD) signals in different brain regions without tasks, has been widely used to investigate different diseases, such as psychosis $(10,11)$, depression (12), Huntington's disease (13), stroke (14), and AD $(15,16)$. During rs-fMRI, participants rest with their eyes closed or focus on a visual fixation while being examined. This is more convenient and less taxing than task-based fMRI or neuropsychological tests for patients with cognitive impairment. Functional connectivity within and between different brain regions can be assessed, and intrinsic brain networks such as the default mode, executive control, visual, salience, and auditory networks can be identified by analyzing BOLD signals (17). These studies suggest that aberrant regional spontaneous fluctuations of BOLD $(18,19)$, functional connectivity (20), and widespread alterations in functional brain network architecture $(21,22)$ could all occur early in AD pathophysiology. Rs-fMRI can also contribute to evaluating the different treatment and detect even subtle changes after a short period of treatment (23). Overall, resting state neuroimaging and other methodologies may provide useful biomarkers for early diagnosis of $\mathrm{AD}$ and understanding the underlying mechanism (24).

In this paper, we review recent progresses of MCI criteria and nomenclature, resting state neuroimaging studies on brain functional activity in patients with MCI, current knowledge of the influences of genetic and educational factors on MCI and the work on diagnosing and classifying MCI. In this review, we focus on the fMRI-based studies and amnestic MCI, but we mention other approaches and MCI with $\mathrm{AD}$ biomarkers/pathologic change as well.

\section{EVOLUTION OF MCI}

MCI was first proposed by Petersen et al. (25) and the diagnosis was mostly depended on clinical or cognitive appearance. After that, many researchers have investigated and expanded the nomenclature. A few criteria $(4,26-28)$ were derived from these studies and MCI was noticed to be heterogeneous and unstable. MCI could be one domain affected or multiple-domain affected and it could be caused by Alzheimer's disease, vascular dementia, depression, or other medical conditions (29). Without general or recommended biomarkers, in a long period, researchers applied different diagnostic criteria to define MCI. The core definitions of amnestic MCI in early criteria are the following: (1) not normal for age; (2) cognitive decline; (3) essentially normal functional activities; (4) not demented; (5) memory impaired. In 2010s, NIA-AA (4) and IWG (3) became more aware of the importance of biomarkers and put more emphasis on $\mathrm{A}$ $\beta$, tau, and other $\mathrm{AD}$ related biomarkers. NIA-AA proposed a new research framework toward AD in 2018, and MCI could either be a syndromal cognitive stage between cognitive normal and dementia which was consistent with the past criteria or be detailed refined according to biomarker profile and cognitive appearance (5). With this new research framework, researchers should enable to target more precise AD-related MCI, and enhance efforts to understand the pathology process of $\mathrm{AD}$ and different etiology of dementia. However, the applications of core $\mathrm{AD}$-related biomarkers are expensive, not necessary to apply for every MCI study. On the other hand, we should develop a lessexpensive and credible biomarker to investigate the MCI, as the population of MCI is even larger than AD. The criteria of MCI were summarized in Table $\mathbf{1 .}$

\section{COGNITIVELY UNIMPAIRED AGING}

To investigate the aberrant brain activity in cognitive impaired old people, changes of brain structure, and function associated with aging should be firstly documented. Raichle (17), Salvador et al. (32), and Achard et al. (33) have demonstrated a sensible, symmetrical architecture of the human brain which could be characterized with a few networks and organized with a small-world network topology. The functional connectivity and structure alteration of brain were associated with memory and aging. Specifically, Ward et al. (34) reported that default mode network (DMN) functional connectivity and hippocampus volume were associated with memory among old individuals. Inter-network functional activity also changed as people aged. Anticorrelated activity between DMN and dorsal attention network was found significantly decreased with age (35). The degrees of functional alteration were different among different brain regions and cognitively normal individuals.

Importantly, AD biomarkers, like amyloid burden, mediate the relationship between age and brain function. Using in vivo amyloid imaging and fMRI, both Hedden et al. (36) and Sperling et al. (37) found aberrant DMN activity in cognitively unimpaired aging with amyloid positive. The patterns of disruption of functional activity were linked to amyloid pathology. Cognitively unimpaired aging with high amyloid burden displayed more reduced functional correlations within posterior cingulate cortex (PCC) and other regions related to memory encoding. Especially, these alterations were stable after controlling for age and structural atrophy which suggested clinically normal older people with disrupted functional activity, particularly with biomarkers of $\mathrm{AD}$, were the susceptible population of AD. Notably, the relationship between amyloid burden and functional connectivity is complex and may be biphasic or multi-phasic changes across the longitudinal process of Alzheimer's continuum. By way of example, Lim et al. (38) reported that greater DMN functional connectivity in cognitively 


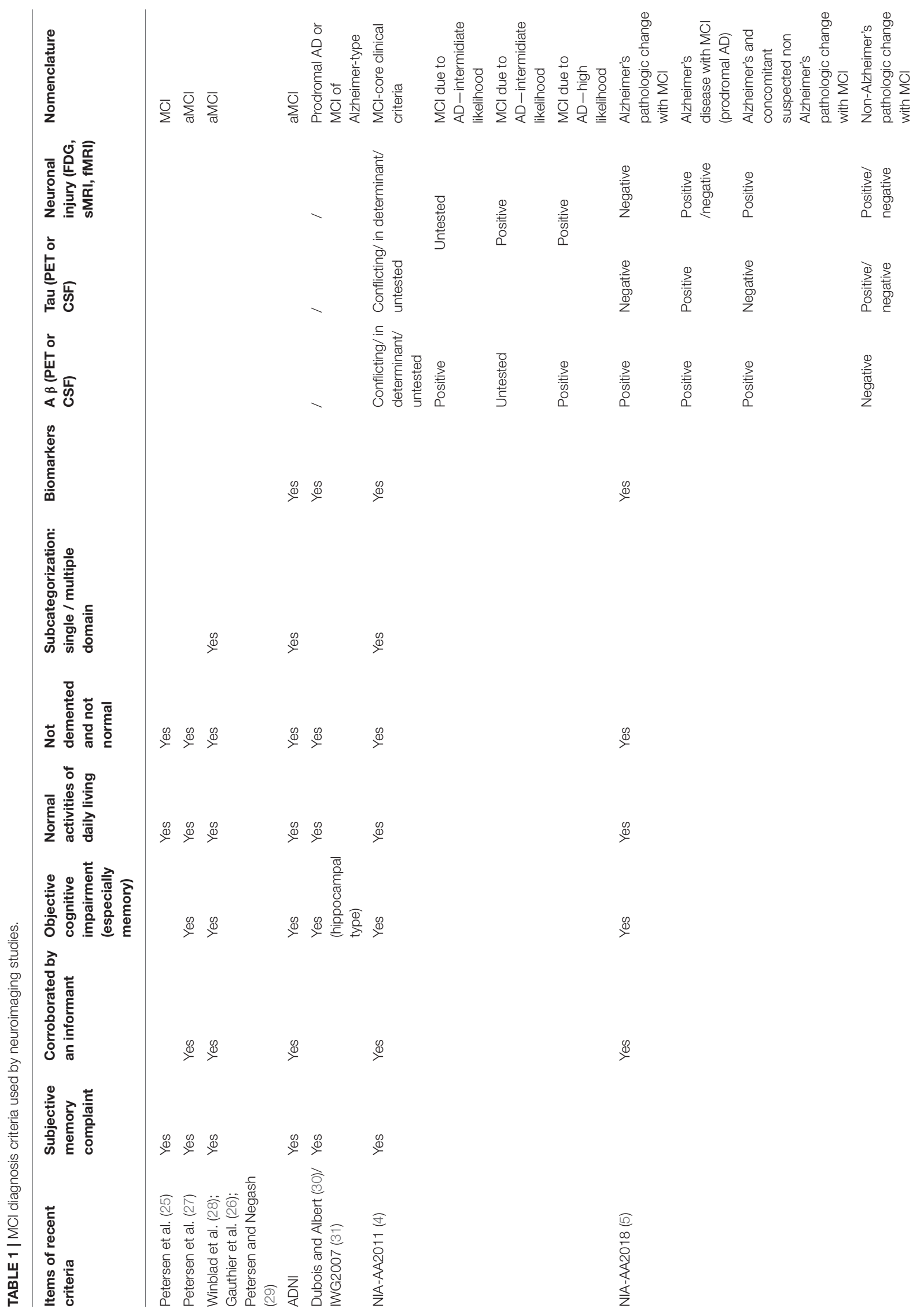


unimpaired aging with positive amyloid burden compared with those with negative amyloid burden. For defending the hazards of amyloid pathology, the brain may develop a response with compensatory higher functional connectivity in some brain regions (39).

\section{MILD COGNITIVE IMPAIRMENT}

Recent studies have demonstrated distinct intrinsic functional brain network architectures in patients with various neurodegenerative diseases, and with each disease having a distinct abnormal brain activity pattern $(40,41)$. The MCI patients were characterized with disruption of functional connectivity within DMN $(19,42,43)$ which involved the ventral medial prefrontal cortex, dorsal medial prefrontal cortex, PCC, adjacent precuneus, and lateral parietal cortex, indicating brain activity as a potential target of diagnose. Regional homogeneity (ReHo) (44), amplitude of low-frequency fluctuation (ALFF) (45), functional connectivity analysis as well as graph theory have been used to investigate the underlying pathological process of MCI.

\section{ReHo and ALFF}

ReHo assumes that a given voxel is temporally similar to that of its neighbors and voxels within a functional brain area are more temporally homogeneous when this is involved in a specific condition. ALFF is found highly synchronous among functional brain systems in normal subjects and less synchronous in patients with mental diseases. Changes in the resting state brain activity of MCI have been evaluated by ReHo or ALFF of BOLD signals, as illustrated in Figure 1. Aberrant ReHo was found prefrontal cortex, bilateral posterior cingulate gyrus/precuneus and inferior parietal lobule in patients with MCI, compared with normal aging $(19,46)$. Similar abnormal functional patterns were reported in ALFF studies of MCI (18) and these patterns exhibited different spatial patterns depending on various frequency bands (42). Both increased and decreased activity could coexist in MCI. The decreased activity in the cuneus/precuneus was found to be associated with reduced memory performance (18) and the increased activity could be a compensation for damage by recruitment of other regions. However, as MCI is a heterogeneous state, AD biomarker related approaches and detailed cognitive examinations may bear more fruit. A recent study combing rs-fMRI and CSF showed the ReHo value was associated with $A \beta$ level in superior temporal gyrus in single domain MCI while the multiple-domain MCI exhibited a more complex pattern of pathology and functional activity (47).

\section{Functional Connectivity}

Resting state functional connectivity describes temporal correlations of BOLD signal between different brain regions/voxels and is the basis of graph theory analysis. Independent component analysis (ICA) and seed-based analysis are the two main methods to analyze the functional connectivity. Early rs-fMRI studies have shown impaired functional connectivity within DMN or between different networks in patients with MCI $(22,48)$. Subsequent studies revealed decreased functional activity in the DMN regions and increased functional activity in the frontal cortex and other regions (Figure 2) (48-50), which indicated that the disrupted and compensatory patterns were general in MCI patients and could be detected by different resting state analysis. Similarly, considering the methodological differences or clinical heterogeneity or biphasic changes across the longitudinal process, the degree of alteration of functional connectivity in the hippocampus and PCC was consistent with poor performance of neuropsychological tests in MCI, indicating the potential of functional connectivity as a biomarker of cognitive preditor (51-53).

In addition to the alteration of DMN in MCI, which has been widely reported, altered functional connectivity within, and/or between different other brain networks such as the executive control, salience, dorsal attention, and sensory-motor networks may also occur in MCI (54-56). For instance, Brier et al. (56) recruited 510 participants with CDR (clinical dementia rating) ranging from 0 to 1 to investigate five functional brain networks through rs-fMRI. They found decreased connectivity within all networks and decreased anti-correlations between these networks as CDR increased. Specifically, some networks were preferentially affected at certain CDR stages. Similarly, Esposito et al. (35) suggested that anti-correlation between DMN and DAN decreased with age, but such reduction was more significant in patients with MCI. Furthermore, with regard to whether such functional changes were generated by aging or pathology process of $\mathrm{AD}$, Brier et al. (57) examined two groups cognitively normal people with or without AD-related CSF biomarkers. Their results suggested that $\mathrm{AD}$ pathology accounted for a large portion of the alterations of functional connectivity. It is still not fully understood how and when elevated AD pathology affects functional connectivity, but these studies indicate the possibility of finding out the relationship between functional connectivity and AD pathology, which could help to establish a safer and/or less-expensive approach for targeting pre-dementia population.

\section{Graph Theory}

Graph theory has been widely used to analyze the organization of the societies, information networks, and internet, but has rather limited use in neuroscience until now. Recent studies have applied this approach to investigate the brain's topological organization $(32,33)$ that revealed disrupted topological organization of the whole brain in MCI $(58,59)$. Based on rs-fMRI, patients with MCI showed decreased overall global functional connectivity of brain connectome and the hubs and important connections between DMN and other functional systems were impaired within MCI patients (58). Such disruption could be partially verified or caused by structural loss of neural fibers, such as corpus callosum, as detected by DTI (60). In addition, as the graph theory analysis assesses the brain activity as a whole, it results in different and new insights of understanding the mechanism of MCI. Zhang et al. explored the connectome in MCI with two dimensions. They reported that both local and remote connectivity dysfunctions were detected in MCI with rs-fMRI while episodic memory performance in MCI was 


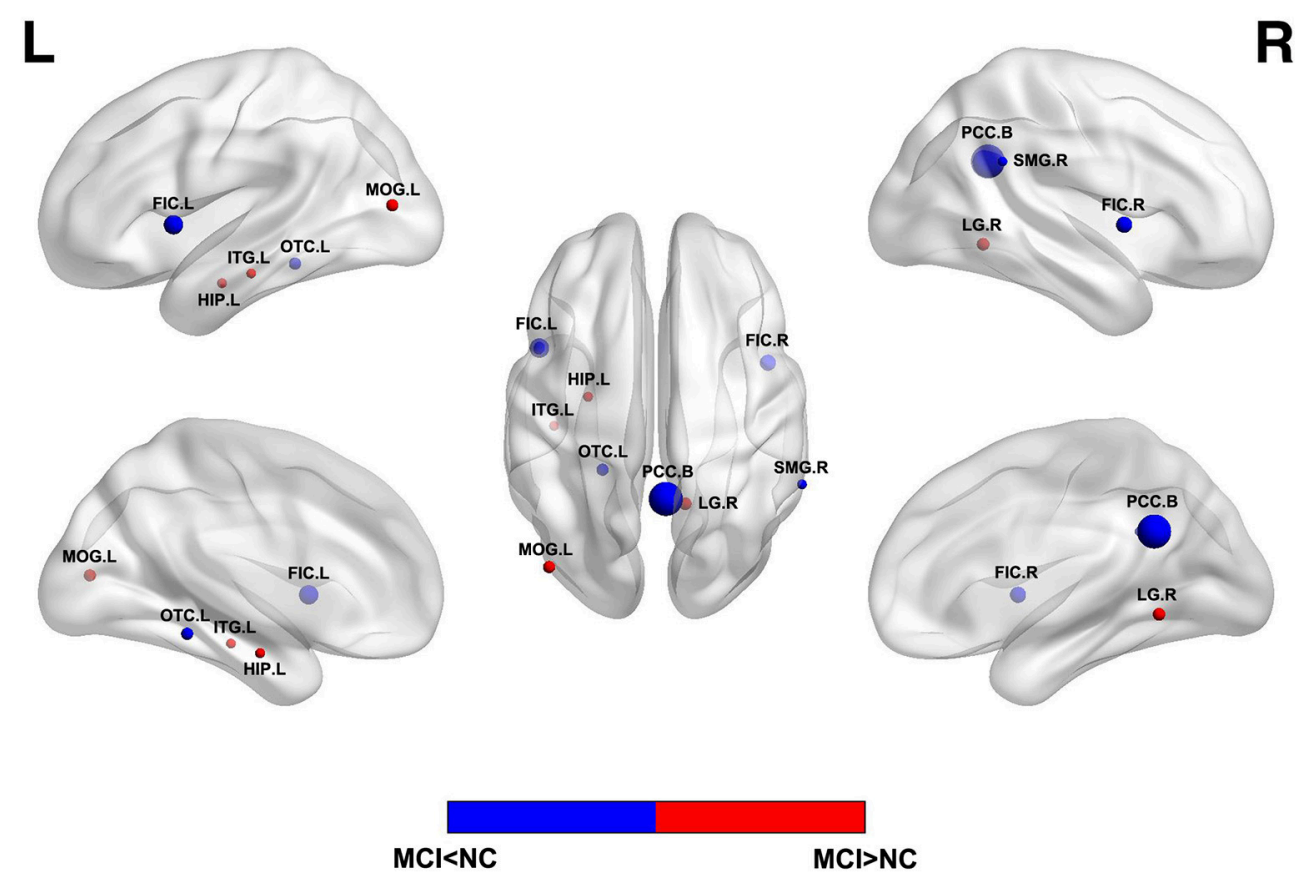

FIGURE 1 | Abnormal regional functional activity in $\mathrm{MCl}$ patients compared with normal controls. Blue = lower functional activity in $\mathrm{MCl}$ patients vs. normal controls, and red = higher functional activity in $\mathrm{MCl}$ patients vs. normal controls. The sizes of balls represent the relative areas of abnormality in these brain regions. $\mathrm{L}$, left; R, right; B, both hemispheres; FIC, frontoinsular cortex; OTC, occipitotemporal cortex; SMG, supramarginal gyrus; LG, lingual gyrus; MOG, middle occipital gyrus; HIP, hippocampus; ITG, inferior temporal gyrus.
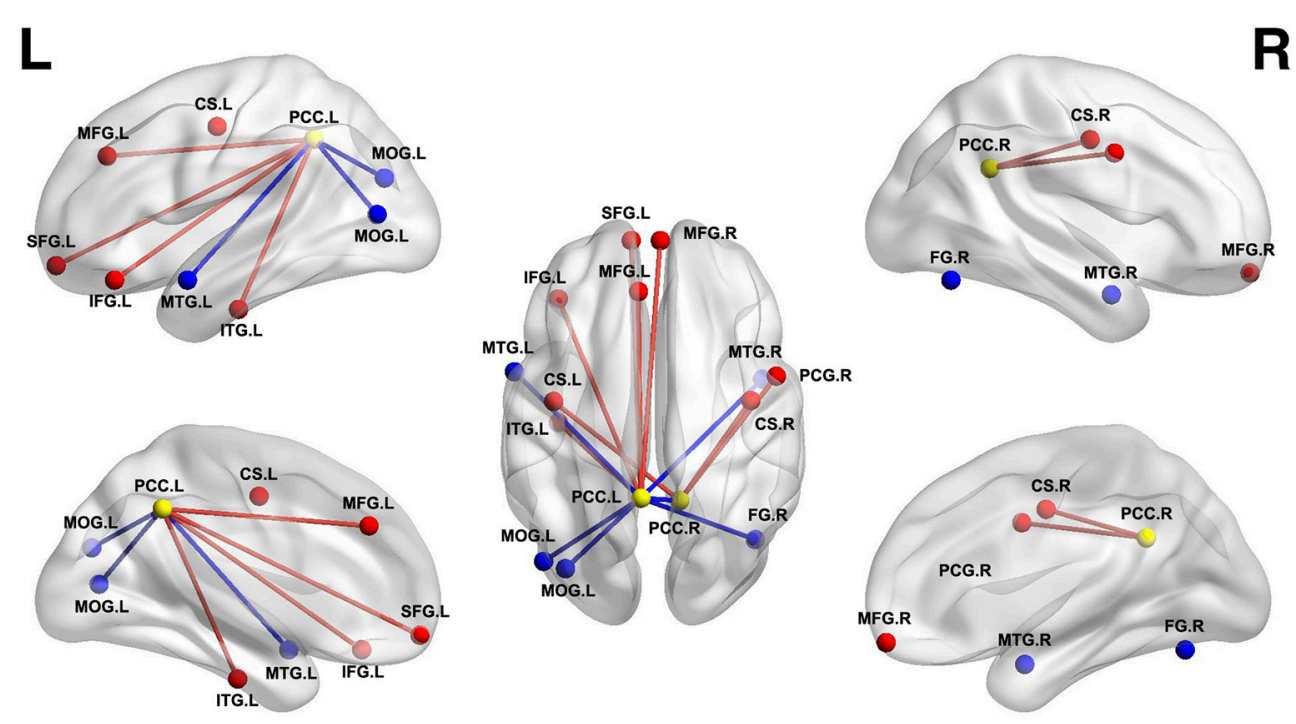

\section{$\mathbf{R}$}

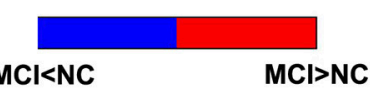

FIGURE 2 | Abnormal functional connectivity between PCC and other brain regions in MCl patients compared with normal controls. Blue = lower functional connectivity in $\mathrm{MCl}$ patients vs. normal controls, and red = higher functional connectivity in MCl patients vs. normal controls. L, left; R, right; MOG, middle occipital gyrus; MTG, middle temporal gyrus; FG, fusiform gyrus; SFG, superior frontal gyrus; MFG, medial frontal gyrus; IFG, inferior frontal gyrus; ITG, inferior temporal gyrus; CS, central sulcus; PCG, precentral gyrus. 
only associated with remote connectivity within DMN. Liu et al. (61) found abnormal rightward laterality in MCI compared with normal controls, which indicated a similar compensatory mechanism detected with above rs-fMRI analysis but displayed in a different appearance. Further, because these changes in functional connectome were correlated with patients' memory performance, graph theory analysis could also act as a method of differentiating individuals with MCI from normal aging (58).

\section{Multi-Modal Imaging Study}

Although rs-fMRI is one of the convenient and noninvasive imaging modality to study pathophysiology of MCI, combination with other techniques can investigate both abnormal structural and functional features as well as their relations in MCI, improve the quality of related clinical trial and reduce requirement of sample sizes $(62,63)$. Multi-modal imaging studies have confirmed correlations between structural and functional alterations $(63,64)$. Collecting gray matter volumes and functional connectivity information simultaneously could serve as a better indicator for predicting the cognitive deficits in MCI (65). In a recent multi-modal study, a novel PET/fMRI scanner was applied to normal aging, patients with MCI and AD to evaluate the resting-state brain glucose metabolism and brain functional activity (64). The metrics [ReHo, fractional ALFF and group ICA with dual regression(gICA-DR)] of rs-fMRI were showed to be correlated with glucose metabolism among all three groups, but the degree of correlation reduced $17 \%$ in $\mathrm{MCI} / \mathrm{AD}$ groups. With the progression of the disease or higher amyloid levels, MCI patients showed severer hippocampus atrophy, reduced structural connectivity of the corpus callosum, and lower functional connectivity between hippocampus and precuneus and higher hippocampal metabolism and amplitude of delta rhythms at rest $(66,67)$. Although criteria and investigators have paid more attention on $\mathrm{AD}$ biomarker profile in the last decade, the number of multi-modal studies and participants is still small compared with that of single modal studies. To address this, combing rs-fMRI with AD biomarker examinations and multi-center collaborations should be more stressed.

\section{EARLY DIAGNOSIS AND CONVERSION OF $\mathrm{MCI}$}

\section{Early Diagnosis of $\mathrm{MCl}$}

Early diagnosis of MCI is important for timely and potentially successful therapeutic intervention. Although substantial research has been dedicated in the last decades, identification of patients with MCI who are at risk of $\mathrm{AD}$ is still a challenge. Resting state neuroimaging is an efficient and powerful technique that facilitates the discovery of abnormalities in MCI patients for the consistency among amounts of studies. Various data processing methods have been used to diagnose or classify MCI based on rs-fMRI scans. Here, some main methods for achieving high diagnostic power for MCI are summarized in Table 2.

Most studies for classification of MCI through rs-fMRI were based on functional connectivity or network architecture features between normal individuals and patients. Some of them focused on the key network of MCI, distinguishing MCI from normal controls based on DMN brain activity of rsfMRI (68). Others depended on the whole-brain activity (69), calculating functional connectivity between various regions (70), or using the graph theory metrics (71). Since the course of $\mathrm{AD}$ is continuous, the patients in $\mathrm{AD}$ continuum share many common features. On one hand, it's good for investigators to identify patients with MCI from normal aging; on the other hand, it becomes more difficult to differentiate between MCI and other stages. For example, one study analyzed DMN brain activity by using both volume of interest (VOI)-based signal time course and independent component analyses (ICA) for identifying patients with dementia (68). They found $82 \%$ patients with MCI contained the characteristics of AD patients. Chen et al. applied a large-scale network analysis to classify normal aging and patients with MCI and AD (70). The AUC analysis yielded 95\% classification power, 93\% sensitivity, and 90\% specificity between patients with MCI and normal controls.

Great efforts have been made to improve the algorithms of classification $(72,73)$. Instead of simply correlating pairwise regional activities, recent studies used different approaches to construct correlation networks to simulate the actual biological networks of brain. By combining a graph theoretical approach with machine learning methods, Khazaee and colleagues achieved $88.4 \%$ classification accuracy of AD, MCI, and controls. Such approach used the optimal features extracted from graph measures by a support vector machine (SVM) (71). Chen et al. (72) proposed a model of constructing a high-order rsfMRI functional connectivity network by grouping correlations for every pair of brain regions into different clusters, whose respective mean correlation time series were represented as highorder correlations among different brain regions. Other methods and models by analogy with biological connectivity in the human brain have also been proposed $(73,74)$. Moreover, a recent fMRI study not only investigated the temporal properties, but also focused on the temporal variability of functional connectivity between specific brain regions (75). As the development of 7TMRI and improvement of algorithms of MRI, using spatiotemporal interaction patterns of brain activity to classify patients and normal controls and achieving more accuracy of classification will be easier in future.

To avoid the shortcomings of single neuroimaging modality and/or target the MCI patients in AD continuum, recent studies applied multi-modal combinations of sMRI, rs-fMRI, and DTI to classify MCI from controls. Wee et al. integrated diffusion tensor imaging (DTI) and rs-fMRI with multi-kernel SVM to improve the classification of $\mathrm{AD}, \mathrm{MCI}$, and normal aging (76). This approach yielded 95\% AUC, 100\% sensitivity, and 94\% specificity between patients with MCI and normal controls. Another similar neuroimaging study also yielded high classification accuracy, at more than 95\% (77). Thus, multimodal connectivity networks have yielded better results in identifying patients with MCI. Together, methods derived from the interactions among different functional networks or derived from multi-modal are better than that derived from a single network or one modal. However, we should bear in mind that many recent resting state studies used different criteria and approaches to diagnosis MCI, future 
TABLE 2 | Summary of studies using different models for early diagnosis of AD.

\begin{tabular}{|c|c|c|c|c|c|c|c|c|}
\hline Papers & $\mathrm{MCl}$ & Control & Imaging modalities & Data analysis & Acc. (\%) & Sen. (\%) & Spec. (\%) & Auc. (\%) \\
\hline Koch et al. (68) & 17 & 21 & $\mathrm{fMRl}$ & ICA \& VOI-based time course & 81.6 & 64.7 & 95.2 & - \\
\hline Qian et al. (69) & 37 & 32 & fMRI & CEEMD \& SVM & 93.3 & - & - & 94.1 \\
\hline Chen et al. (70) & 15 & 20 & fMRI & LSN \& LDA & 91.0 & 93.0 & 90.0 & 95.0 \\
\hline Khazaee et al. (71) & 89 & 45 & $\mathrm{fMRl}$ & SVM & 72.0 & 84.9 & 61.5 & - \\
\hline Chen et al. (72) & 29 & 30 & fMRI & LASSO \& SVM & 88.1 & 86.2 & 90.0 & 93.0 \\
\hline Yu et al. (73) & 50 & 49 & fMRI & WSGR & 84.8 & 86.8 & 72.1 & 86.8 \\
\hline Challis et al. (74) & 50 & 39 & fMRI & GP-LR & 75.0 & $100 \%$ & 50.0 & 70.0 \\
\hline Jie et al. (75) & 99 & 50 & fMRI & SVM & 78 & 82 & 74 & 77 \\
\hline Wee et al. (76) & 10 & 17 & DTI \& fMRI & SVM & 96.3 & 100 & 94.1 & 95.3 \\
\hline Zhu et al. (77) & 22 & 22 & DTI \& fMRI & CFS \& SVM & 95.4 & 95.0 & 95.9 & - \\
\hline
\end{tabular}

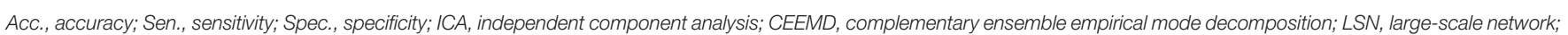

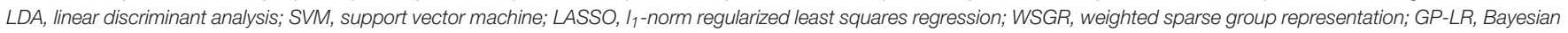
Gaussian process logistic regression; CFS, correlation-based feature selection.

research is required to replicate these findings and it is too soon to make a conclusion about the best algorithm of classification.

\section{Conversion of $\mathrm{MCl}$}

As a transient stage of cognitive stage, patients with MCI are likely to convert to $\mathrm{AD}$, especially those with $\mathrm{AD}$ biomarkers. The rate of conversion of patients with MCI older than 65 years to AD when followed for 2 years was about 14.9\% (9). Equally important to MCI's etiology, knowledge of the mechanism of conversion from $\mathrm{MCI}$ to $\mathrm{AD}$ can help to invent new treatments to slow or halt disease progression and modify available therapies. To date, neuroimaging studies have provided some clues of how MCI is converted to $\mathrm{AD}$. Li et al. (78) tracked MCI patients who had neurocognitive tests and rs-fMRI before and after the conversion. The results showed a significantly lower functional connectivity between the left angular gyrus and middle occipital gyrus in the converters than non-converters. Another study reported both intra- and inter-network longitudinal disruptions in MCI, the decline of compensatory ability may act as the potential cause of conversion (79). Consistent with abnormal functional connectivity, study of the DTI imaging modality showed selective and progressive disruptions of structural connectivity in patients with MCI that could be used to separate converters from nonconverters (80). As compared to the above studies which had similar small-moderate sample sizes, Buckley et al. (81) analyzed 237 clinically normal older adults' longitudinal data with amyloid imaging and rs-fMRI. They speculated that lower functional connectivity predicted more rapid cognitive decline and amyloid burden would accelerate this process. In addition, they suggested rs-fMRI may act as a predictor of early $\mathrm{AD}$-related cognitive decline. In summary, there is no current consensus on how the MCI converts to next stage, and the approaches used exhibit vast heterogeneity.

\section{INFLUENCES OF GENES AND EDUCATION}

\section{Influences of Genetics}

Increasing evidence suggest that multiple factors, including diabetes, obesity, physical and mental inactivity, depression, smoking, low educational attainment, and genetics all may play a role in $\mathrm{AD}$. Among them, genetic association with $\mathrm{AD}$ is strong (82). Previous studies have revealed that genes like ATP-binding cassette transporter A7 (ABCA7), clusterin (CLU), complement receptor 1 (CR1), apolipoprotein Eع4 (APOE4), phosphatidylinositol-binding clathrin assembly protein (PICALM), are associated with $\mathrm{AD}$ risk. The identification of these genetic loci and their mechanism of pathological function are crucial for understanding the etiology of $\mathrm{AD}$ and for early diagnosis. Combined neuroimaging-genetic studies can provide crucial evidence of genetic effects on brain functioning of MCI.

APOE4 is one of the major genetic risk factor for $\mathrm{AD}$ and is involved in lipid homeostasis in the brain that influences multiple neurophysiological pathways and MCI network dysfunction (83). Study of APOE4 showed a strong overlap in decreased functional connectivity between patients with MCI and APOE4 carriers with or without MCI (84). Only patients with MCI had decreased functional connectivity of prefrontal cortical areas compared with APOE4 carriers, suggesting that the etiology of MCI has multiple factors besides genetics. The results of another combined APOE4rs-fMRI study showed that APOE4 may accelerate functional connectivity decline in related brain networks in patients with MCI (85). Conversely, APOE2 would produce agedependent and divergent effect on functional connectivity (86).

Additionally, other metabolic pathways may also play a role in pathogenesis of MCI. CLU has been associated with brain atrophy (87). Bai et al. (88) correlated the results of CLU genotyping and rs-fMRI imaging in patients with MCI and normal controls, and found significant effects of the CLU CC genotype on cortical midline regions, especially task-positive networks in MCI. In a later study with similar methods, Bai et al. (89) found the promoter haplotypes of IL-10 may be associated with abnormal functional communications in the left hippocampal-frontoparietal cortex in MCI. Another inflammation-related gene, Interleukin 1 beta (IL-1 $\beta$ ), was associated with abnormal ALFF in left parietal cortex, bilateral 
frontal cortex, and left occipital cortex in patients with MCI (90). PICALM gene polymorphisms were found to interact with the functional activity in the left middle temporal gyrus and left middle frontal gyrus (91). In addition, functional connectivity in the gene-related regions may be correlated with memory performance for a distinct genotype subgroup of patients with MCI $(86,91)$. Although it is clear for investigators that these genes may play a role in MCI pathogenesis, it's easy to be ignored the genes are originally functional in normal people. For instance, Seddighi et al. (92) suggested that low expression of SPARCL1 were associated with accelerated memory loss, reduced brain volumes, and decreased cerebral perfusion during aging. Future research should attempt to specify the interaction of different genetypes with pathology process in MCI with $\mathrm{AD}$ biomarkers.

\section{Influences of Cognitive Reserve}

Recent work on the functional connectivity of the DMN and other related resting-state networks in patients with MCI suggested the coexistence of impairment and compensation. A factor called cognitive reserve (CR), which is often assessed via years of education and IQ, has attracted attention for its relationships to cognitive performance and level of brain damage. Studies suggested the attempts by the patients' brains to compensate for damage by using the existing faculties of functional cognitive brain regions. Neuroimaging studies show CR modulates functional connectivity in large-scale network in patients with MCI and that is correlated with memory scores (93-95). Serra et al. (93) reported that high CR in patients with MCI was associated with increased functional connectivity in a network of fronto-parietal nodes and decreased functional connectivity in a network of fronto-temporo-cerebellar nodes. A recent study of functional connectivity between the DMN and the DAN, which is associated with cognitive control and working memory, suggested that negative DMN-DAN correlation was associated with poorer memory performance in patients with MCI. However, after adjusting for the CR, this association could be compensated by a higher CR (95). Not surprisingly, rs-fMRI could conversely be used to predict the significance of CR in patients with MCI (96). However, since the cognitive function declines with age, whether the changes observed are due to the normal aging or the pathological process of $\mathrm{AD}$ should be considered.

\section{REFERENCES}

1. McDade E, Bateman RJ. Stop Alzheimer's before it starts. Nature (2017) 547:153-5. doi: 10.1038/547153a

2. Scheltens P, Blennow K, Breteler MM, de Strooper B, Frisoni GB, Salloway S, et al. Alzheimer's disease. Lancet (2016) 388:505-17. doi: 10.1016/S0140-6736(15)01124-1

3. Dubois B, Feldman HH, Jacova C, Hampel H, Molinuevo JL, Blennow K, et al. Advancing research diagnostic criteria for Alzheimer's disease: the IWG2 criteria. Lancet Neurol. (2014) 13:614-29. doi: 10.1016/S1474-4422(14)70 090-0

4. Albert MS, DeKosky ST, Dickson D, Dubois B, Feldman HH, Fox NC, et al. The diagnosis of mild cognitive impairment due to Alzheimer's disease:

\section{FUTURE WORK AND CONCLUSION}

As seen from the studies presented here, rs-fMRI has already contributed substantially to the field of MCI's research. Alteration of functional brain activity within DMN and between other networks have been detected with multiple analysis in cognitive impaired people, and such changes are accelerated by MCI related pathology process and other risk factors. As an intermediate stage of $\mathrm{AD}$ continuum, MCI patients reserve the capability of compensating the detriment of the disease. The possible compensatory mechanisms and how to differentiate the $\mathrm{MCI}$ due to $\mathrm{AD}$ from the pre-dementia are still a mystery. Longitudinal study design and improvement of data analysis would help to solve these problems. In future research, it will be important to determine which features in MCI are the signs of progression to $\mathrm{AD}$. As imaging modalities improve, multi-center collaboration, and data exchanges expand, and as MCI criteria are more clearly delineated, we may expect further refinement in characterizing the association between MCI and brain and/or cognition based biomarkers.

\section{AUTHOR CONTRIBUTIONS}

LL, GX, and YH contributed conception and design of the study. LL wrote the first draft of the manuscript. All authors contributed to manuscript revision, read, and approved the submitted version.

\section{FUNDING}

This article was supported by The National Key Research and Development Program of China (2016YFC1306300), National Natural Science Foundation of China (Grant 61633018), Beijing Municipal Commission of Health and Family Planning (PXM2018_026283_000002).

\section{ACKNOWLEDGMENTS}

The authors gratefully acknowledge all participants for their enduring collaboration and interest in this research. Also, the authors would like to thank the staff from the Center of Alzheimer's Disease of the Beijing Institute for Brain Disorders and everyone else who helped out with various issues.

recommendations from the National Institute on Aging-Alzheimer's Association workgroups on diagnostic guidelines for Alzheimer's disease. Alzheimer's Dement. (2011) 7:270-9. doi: 10.1016/j.jalz.2011. 03.008

5. Jack CR Jr, Bennett DA, Blennow K, Carrillo MC, Dunn B, Haeberlein SB, et al. NIA-AA research framework: toward a biological definition of Alzheimer's disease. Alzheimer's Dement. (2018) 14:535-62. doi: 10.1016/j.jalz.2018.02.018

6. Roberts R, Knopman DS. Classification and epidemiology of MCI. Clin Geriatr Med. (2013) 29:753-72. doi: 10.1016/j.cger.2013.07.003

7. Ward A, Tardiff S, Dye C, Arrighi HM. Rate of conversion from prodromal Alzheimer's disease to Alzheimer's dementia: a systematic review of the literature. Dement Geriatr Cogn Dis Extra (2013) 3:320-32. doi: $10.1159 / 000354370$ 
8. Alzheimer's A. 2016 Alzheimer's disease facts and figures. Alzheimer's Dement. (2016) 12:459-509. doi: 10.1016/j.jalz.2016.03.001

9. Petersen RC, Lopez O, Armstrong MJ, Getchius TSD, Ganguli M, Gloss D, et al. Practice guideline update summary: mild cognitive impairment: report of the guideline development, dissemination, and implementation subcommittee of the american academy of neurology. Neurology (2018) 90:126-35. doi: 10.1212/WNL.0000000000004826

10. Moran LV, Tagamets MA, Sampath H, O'Donnell A, Stein EA, Kochunov P, et al. Disruption of anterior insula modulation of largescale brain networks in schizophrenia. Biol Psychiatry (2013) 74:467-74. doi: 10.1016/j.biopsych.2013.02.029

11. Woodward ND, Heckers S. Mapping thalamocortical functional connectivity in chronic and early stages of psychotic disorders. Biol Psychiatry (2016) 79:1016-25. doi: 10.1016/j.biopsych.2015.06.026

12. Kaiser RH, Andrews-Hanna JR, Wager TD, Pizzagalli DA. Large-scale network dysfunction in major depressive disorder: a meta-analysis of resting-state functional connectivity. JAMA Psychiatry (2015) 72:603-11. doi: 10.1001/jamapsychiatry.2015.0071

13. Harrington DL, Rubinov M, Durgerian S, Mourany L, Reece C, Koenig K, et al. Network topology and functional connectivity disturbances precede the onset of Huntington's disease. Brain (2015) 138 (Pt 8):2332-46. doi: 10.1093/brain/awv145

14. Baldassarre A, Ramsey L, Rengachary J, Zinn K, Siegel JS, Metcalf NV, et al. Dissociated functional connectivity profiles for motor and attention deficits in acute right-hemisphere stroke. Brain (2016) 139(Pt 7):2024-38. doi: 10.1093/brain/aww107

15. Sheline YI, Raichle ME. Resting state functional connectivity in preclinical Alzheimer's disease. Biol Psychiatry (2013) 74:340-7. doi: 10.1016/j.biopsych.2012.11.028

16. Dennis EL, Thompson PM. Functional brain connectivity using fMRI in aging and Alzheimer's disease. Neuropsychol Rev. (2014) 24:49-62. doi: 10.1007/s11065-014-9249-6

17. Raichle ME. The brain's default mode network. Annu Rev Neurosci. (2015) 38:433-47. doi: 10.1146/annurev-neuro-071013-014030

18. Pan P, Zhu L, Yu T, Shi H, Zhang B, Qin R, et al. Aberrant spontaneous low-frequency brain activity in amnestic mild cognitive impairment: a metaanalysis of resting-state fMRI studies. Ageing Res Rev. (2017) 35:12-21. doi: 10.1016/j.arr.2016.12.001

19. Zhang Z, Liu Y, Jiang T, Zhou B, An N, Dai H, et al. Altered spontaneous activity in Alzheimer's disease and mild cognitive impairment revealed by Regional Homogeneity. Neuroimage (2012) 59:1429-40. doi: 10.1016/j.neuroimage.2011.08.049

20. Jacobs HI, Wiese S, van de Ven V, Gronenschild EH, Verhey FR, Matthews PM. Relevance of parahippocampal-locus coeruleus connectivity to memory in early dementia. Neurobiol Aging (2015) 36:618-26. doi: 10.1016/j.neurobiolaging.2014.10.041

21. Minati L, Chan D, Mastropasqua C, Serra L, Spano B, Marra C, et al. Widespread alterations in functional brain network architecture in amnestic mild cognitive impairment. J Alzheimer's Dis. (2014) 40:213-20. doi: 10.3233/JAD-131766

22. Sorg C, Riedl V, Muhlau M, Calhoun VD, Eichele T, Laer L, et al. Selective changes of resting-state networks in individuals at risk for Alzheimer's disease. Proc Natl Acad Sci USA. (2007) 104:18760-5. doi: 10.1073/pnas.0708803104

23. Canu E, Sarasso E, Filippi M, Agosta F. Effects of pharmacological and nonpharmacological treatments on brain functional magnetic resonance imaging in Alzheimer's disease and mild cognitive impairment: a critical review. Alzheimer's Res Ther. (2018) 10:21. doi: 10.1186/s13195-018-0 347-1

24. Frisoni GB, Boccardi M, Barkhof F, Blennow K, Cappa S, Chiotis $\mathrm{K}$, et al. Strategic roadmap for an early diagnosis of Alzheimer's disease based on biomarkers. Lancet Neurol. (2017) 16:661-76. doi: 10.1016/S1474-4422(17)30159-X

25. Petersen RC, Smith GE, Waring SC, Ivnik RJ, Tangalos EG, Kokmen E. Mild cognitive impairment: clinical characterization and outcome. Arch Neurol. (1999) 56:303-8. doi: 10.1001/archneur.56.3.303

26. Gauthier S, Reisberg B, Zaudig M, Petersen RC, Ritchie K, Broich $\mathrm{K}$, et al. Mild cognitive impairment. Lancet (2006) 367:1262-70, doi: 10.1016/S0140-6736(06)68542-5
27. Petersen RC, Doody R, Kurz A, Mohs RC, Morris JC, Rabins PV, et al. Current concepts in mild cognitive impairment. Arch Neurol. (2001) 58:1985-92. doi: 10.1001/archneur.58.12.1985

28. Winblad B, Palmer K, Kivipelto M, Jelic V, Fratiglioni L, Wahlund LO, et al. Mild cognitive impairment-beyond controversies, towards a consensus: report of the International Working Group on Mild Cognitive Impairment. $J$ Intern Med. (2004) 256:240-6. doi: 10.1111/j.1365-2796.2004.01380.x

29. Petersen RC, Negash S. Mild cognitive impairment: an overview. CNS Spectr. (2008) 13:45-53. doi: 10.1017/S1092852900016151

30. Dubois B, Albert ML. Amnestic MCI or prodromal Alzheimer's disease? Lancet Neurol. (2004) 3:246-8. doi: 10.1016/S1474-4422(04)00710-0

31. Dubois B, Feldman HH, Jacova C, Dekosky ST, Barberger-Gateau P, Cummings J, et al. Research criteria for the diagnosis of Alzheimer's disease: revising the NINCDS-ADRDA criteria. Lancet Neurol. (2007) 6:734-46. doi: 10.1016/S1474-4422(07)70178-3

32. Salvador R, Suckling J, Coleman MR, Pickard JD, Menon D, Bullmore E. Neurophysiological architecture of functional magnetic resonance images of human brain. Cereb Cortex (2005) 15:1332-42. doi: 10.1093/cercor/bhi016

33. Achard S, Salvador R, Whitcher B, Suckling J, Bullmore E. A resilient, low-frequency, small-world human brain functional network with highly connected association cortical hubs. J Neurosci. (2006) 26:63-72. doi: 10.1523/JNEUROSCI.3874-05.2006

34. Ward AM, Mormino EC, Huijbers W, Schultz AP, Hedden T, Sperling RA. Relationships between default-mode network connectivity, medial temporal lobe structure, and age-related memory deficits. Neurobiol Aging (2015) 36:265-72. doi: 10.1016/j.neurobiolaging.2014.06.028

35. Esposito R, Cieri F, Chiacchiaretta P, Cera N, Lauriola M, Di Giannantonio $\mathrm{M}$, et al. Modifications in resting state functional anticorrelation between default mode network and dorsal attention network: comparison among young adults, healthy elders and mild cognitive impairment patients. Brain Imaging Behav. (2018) 12:127-41. doi: 10.1007/s11682-017-9686-y

36. Hedden T, Van Dijk KR, Becker JA, Mehta A, Sperling RA, Johnson $\mathrm{KA}$, et al. Disruption of functional connectivity in clinically normal older adults harboring amyloid burden. J Neurosci. (2009) 29:12686-94. doi: 10.1523/JNEUROSCI.3189-09.2009

37. Sperling RA, Laviolette PS, O'Keefe K, O'Brien J, Rentz DM, Pihlajamaki $\mathrm{M}$, et al. Amyloid deposition is associated with impaired default network function in older persons without dementia. Neuron (2009) 63:178-88. doi: 10.1016/j.neuron.2009.07.003

38. Lim HK, Nebes R, Snitz B, Cohen A, Mathis C, Price J, et al. Regional amyloid burden and intrinsic connectivity networks in cognitively normal elderly subjects. Brain (2014) 137(Pt 12):3327-38. doi: 10.1093/brain/awu271

39. Mormino EC, Smiljic A, Hayenga AO, Onami SH, Greicius MD, Rabinovici GD, et al. Relationships between beta-amyloid and functional connectivity in different components of the default mode network in aging. Cereb Cortex (2011) 21:2399-407. doi: 10.1093/cercor/bhr025

40. Seeley WW, Crawford RK, Zhou J, Miller BL, Greicius MD. Neurodegenerative diseases target large-scale human brain networks. Neuron (2009) 62:42-52. doi: 10.1016/j.neuron.2009.03.024

41. Spetsieris PG, Ko JH, Tang CC, Nazem A, Sako W, Peng S, et al. Metabolic resting-state brain networks in health and disease. Proc Natl Acad Sci USA. (2015) 112:2563-8. doi: 10.1073/pnas.1411011112

42. Han Y, Wang J, Zhao Z, Min B, Lu J, Li K, et al. Frequency-dependent changes in the amplitude of low-frequency fluctuations in amnestic mild cognitive impairment: a resting-state fMRI study. Neuroimage (2011) 55:28795. doi: 10.1016/j.neuroimage.2010.11.059

43. Koch K, Myers NE, Gottler J, Pasquini L, Grimmer T, Forster S, et al. Disrupted intrinsic networks link amyloid-beta pathology and impaired cognition in prodromal Alzheimer's disease. Cereb Cortex (2015) 25:4678-88. doi: 10.1093/cercor/bhu151

44. Zang Y, Jiang $\mathrm{T}$, Lu Y, He Y, Tian L. Regional homogeneity approach to fMRI data analysis. Neuroimage (2004) 22:394-400. doi: 10.1016/j.neuroimage.2003.12.030

45. Zang YF, He Y, Zhu CZ, Cao QJ, Sui MQ, Liang M, et al. Altered baseline brain activity in children with ADHD revealed by resting-state functional MRI. Brain Dev. (2007) 29:83-91. doi: 10.1016/j.braindev.2006.07.002

46. Bai F, Zhang Z, Yu H, Shi Y, Yuan Y, Zhu W, et al. Default-mode network activity distinguishes amnestic type mild cognitive impairment from healthy 
aging: a combined structural and resting-state functional MRI study. Neurosci Lett. (2008) 438:111-5. doi: 10.1016/j.neulet.2008.04.021

47. Luo X, Jiaerken Y, Huang P, Xu XJ, Qiu T, Jia Y, et al. Alteration of regional homogeneity and white matter hyperintensities in amnestic mild cognitive impairment subtypes are related to cognition and CSF biomarkers. Brain Imaging Behav. (2018) 12:188-200. doi: 10.1007/s11682-017-9680-4

48. Qi Z, Wu X, Wang Z, Zhang N, Dong $\mathrm{H}$, Yao L, et al. Impairment and compensation coexist in amnestic MCI default mode network. Neuroimage (2010) 50:48-55. doi: 10.1016/j.neuroimage.2009. 12.025

49. Bai F, Watson DR, Yu H, Shi Y, Yuan Y, Zhang Z. Abnormal resting-state functional connectivity of posterior cingulate cortex in amnestic type mild cognitive impairment. Brain Res. (2009) 1302:167-74. doi: 10.1016/j.brainres.2009.09.028

50. Jin M, Pelak VS, Cordes D. Aberrant default mode network in subjects with amnestic mild cognitive impairment using resting-state functional MRI. Magn Reson Imaging (2012) 30:48-61. doi: 10.1016/j.mri.2011. 07.007

51. Binnewijzend MA, Schoonheim MM, Sanz-Arigita E, Wink AM, van der Flier WM, Tolboom N, et al. Resting-state fMRI changes in Alzheimer's disease and mild cognitive impairment. Neurobiol Aging (2012) 33:2018-28. doi: 10.1016/j.neurobiolaging.2011.07.003

52. Wang Z, Liang P, Jia X, Qi Z, Yu L, Yang Y, et al. Baseline and longitudinal patterns of hippocampal connectivity in mild cognitive impairment: evidence from resting state fMRI. J Neurol Sci. (2011) 309:79-85. doi: 10.1016/j.jns.2011.07.017

53. Gardini S, Venneri A, Sambataro F, Cuetos F, Fasano F, Marchi M, et al. Increased functional connectivity in the default mode network in mild cognitive impairment: a maladaptive compensatory mechanism associated with poor semantic memory performance. J Alzheimer's Dis. (2015) 45:45770. doi: 10.3233/JAD-142547

54. Bai F, Shi Y, Yuan Y, Wang Y, Yue C, Teng Y, et al. Altered self-referential network in resting-state amnestic type mild cognitive impairment. Cortex (2012) 48:604-13. doi: 10.1016/j.cortex.2011.02.011

55. Agosta F, Pievani M, Geroldi C, Copetti M, Frisoni GB, Filippi M. Resting state fMRI in Alzheimer's disease: beyond the default mode network. Neurobiol Aging (2012) 33:1564-78. doi: 10.1016/j.neurobiolaging.2011.06.007

56. Brier MR, Thomas JB, Snyder AZ, Benzinger TL, Zhang D, Raichle $\mathrm{ME}$, et al. Loss of intranetwork and internetwork resting state functional connections with Alzheimer's disease progression. J Neurosci. (2012) 32:88909. doi: 10.1523/JNEUROSCI.5698-11.2012

57. Brier MR, Thomas JB, Snyder AZ, Wang L, Fagan AM, Benzinger $\mathrm{T}$, et al. Unrecognized preclinical Alzheimer disease confounds rs-fcMRI studies of normal aging. Neurology (2014) 83:1613-9. doi: 10.1212/WNL.0000000000000939

58. Wang J, Zuo X, Dai Z, Xia M, Zhao Z, Zhao X, et al. Disrupted functional brain connectome in individuals at risk for Alzheimer's disease. Biol Psychiatry (2013) 73:472-81. doi: 10.1016/j.biopsych.2012.03.026

59. Zhang YW, Zhao ZL, Qi Z, Hu Y, Wang YS, Sheng C, et al. Local-to-remote cortical connectivity in amnestic mild cognitive impairment. Neurobiol Aging (2017) 56:138-49. doi: 10.1016/j.neurobiolaging.2017.04.016

60. Vecchio F, Miraglia F, Curcio G, Altavilla R, Scrascia F, Giambattistelli F, et al. Cortical brain connectivity evaluated by graph theory in dementia: a correlation study between functional and structural data. J Alzheimer's Dis. (2015) 45:745-56. doi: 10.3233/JAD-142484

61. Liu H, Zhang L, Xi Q, Zhao X, Wang F, Wang X, et al. Changes in brain lateralization in patients with mild cognitive impairment and Alzheimer's disease: a resting-state functional magnetic resonance study from Alzheimer's disease neuroimaging initiative. Front Neurol. (2018) 9:3. doi: 10.3389/fneur.2018.00003

62. Wolz R, Schwarz AJ, Gray KR, Yu P, Hill DL. Enrichment of clinical trials in $\mathrm{MCI}$ due to AD using markers of amyloid and neurodegeneration. Neurology (2016) 87:1235-41. doi: 10.1212/WNL.0000000000003126

63. Zhou Y, Yu F, Duong TQ. White matter lesion load is associated with resting state functional MRI activity and amyloid PET but not FDG in mild cognitive impairment and early Alzheimer's disease patients. J Magn Reson Imaging (2015) 41:102-9. doi: 10.1002/jmri.24550
64. Marchitelli R, Aiello M, Cachia A, Quarantelli M, Cavaliere C, Postiglione A, et al. Simultaneous resting-state FDG-PET/fMRI in Alzheimer disease: relationship between glucose metabolism and intrinsic activity. Neuroimage (2018) 176:246-58. doi: 10.1016/j.neuroimage.2018.04.048

65. Xie C, Bai F, Yuan B, Yu H, Shi Y, Yuan Y, et al. Joint effects of gray matter atrophy and altered functional connectivity on cognitive deficits in amnestic mild cognitive impairment patients. Psychol Med. (2015) 45:1799_810. doi: $10.1017 /$ S0033291714002876

66. Galluzzi S, Marizzoni M, Babiloni C, Albani D, Antelmi L, Bagnoli C, et al. Clinical and biomarker profiling of prodromal Alzheimer's disease in workpackage 5 of the innovative medicines initiative PharmaCog project: a 'European ADNI study'. J Int Med. (2016) 279:576-91. doi: 10.1111 /joim. 12482

67. Tahmasian M, Pasquini L, Scherr M, Meng C, Forster S, Mulej Bratec $\mathrm{S}$, et al. The lower hippocampus global connectivity, the higher its local metabolism in Alzheimer disease. Neurology (2015) 84:1956-63. doi: 10.1212/WNL.0000000000001575

68. Koch W, Teipel S, Mueller S, Benninghoff J, Wagner M, Bokde AL, et al. Diagnostic power of default mode network resting state fMRI in the detection of Alzheimer's disease. Neurobiol Aging (2012) 33:466-78. doi: 10.1016/j.neurobiolaging.2010.04.013

69. Qian L, Zheng L, Shang Y, Zhang Y, Zhang Y. Intrinsic frequency specific brain networks for identification of MCI individuals using resting-state fMRI. Neurosci Lett. (2018) 664:7-14. doi: 10.1016/j.neulet.2017.10.052

70. Chen G, Ward BD, Xie C, Li W, Wu Z, Jones JL, et al. Classification of Alzheimer disease, mild cognitive impairment, and normal cognitive status with large-scale network analysis based on resting-state functional MR imaging. Radiology (2011) 259:213-21. doi: 10.1148/radiol.10100734

71. Khazaee A, Ebrahimzadeh A, Babajani-Feremi A. Application of advanced machine learning methods on resting-state fMRI network for identification of mild cognitive impairment and Alzheimer's disease. Brain Imaging Behav. (2015) 10:799-817. doi: 10.1007/s11682-015-9448-7

72. Chen X, Zhang H, Gao Y, Wee CY, Li G, Shen D. High-order resting-state functional connectivity network for MCI classification. Hum Brain Mapp. (2016) 37:3282-96. doi: 10.1002/hbm.23240

73. Yu R, Zhang H, An L, Chen X, Wei Z, Shen D. Connectivity strengthweighted sparse group representation-based brain network construction for MCI classification. Hum Brain Mapp. (2017) 38:2370-83. doi: 10.1002/hbm. 23524

74. Challis E, Hurley P, Serra L, Bozzali M, Oliver S, Cercignani M. Gaussian process classification of Alzheimer's disease and mild cognitive impairment from resting-state fMRI. Neuroimage (2015) 112:232-43. doi: $10.1016 /$ j.neuroimage. 2015.02 .037

75. Jie B, Liu M, Shen D. Integration of temporal and spatial properties of dynamic connectivity networks for automatic diagnosis of brain disease. Med Image Anal. (2018) 47:81-94. doi: 10.1016/j.media.2018.03.013

76. Wee CY, Yap PT, Zhang D, Denny K, Browndyke JN, Potter $\mathrm{GG}$, et al. Identification of MCI individuals using structural and functional connectivity networks. Neuroimage (2012) 59:2045-56. doi: 10.1016/j.neuroimage.2011.10.015

77. Zhu D, Li K, Terry DP, Puente AN, Wang L, Shen D, et al. Connectomescale assessments of structural and functional connectivity in MCI. Hum Brain Mapp. (2014) 35:2911-23. doi: 10.1002/hbm.22373

78. Li Y, Wang X, Li Y, Sun Y, Sheng C, Li H, et al. Abnormal restingstate functional connectivity strength in mild cognitive impairment and its conversion to Alzheimer's Disease. Neural Plast. (2016) 2016:4680972. doi: 10.1155/2016/4680972

79. Zhan Y, Ma J, Alexander-Bloch AF, Xu K, Cui Y, Feng Q, et al. Longitudinal study of impaired intra- and inter-network brain connectivity in subjects at high risk for Alzheimer's disease. J Alzheimer's Dis. (2016) 52:913-27. doi: 10.3233/JAD-160008

80. Hahn K, Myers N, Prigarin S, Rodenacker K, Kurz A, Forstl H, et al. Selectively and progressively disrupted structural connectivity of functional brain networks in Alzheimer's disease - revealed by a novel framework to analyze edge distributions of networks detecting disruptions with strong statistical evidence. Neuroimage (2013) 81:96-109. doi: 10.1016/j.neuroimage.2013.05.011 
81. Buckley RF, Schultz AP, Hedden T, Papp KV, Hanseeuw BJ, Marshall G, et al. Functional network integrity presages cognitive decline in preclinical Alzheimer disease. Neurology (2017) 89:29-37. doi: 10.1212/WNL.0000000000004059

82. Karch CM, Goate AM. Alzheimer's disease risk genes and mechanisms of disease pathogenesis. Biol Psychiatry (2015) 77:43-51. doi: 10.1016/j.biopsych.2014.05.006

83. Reinvang I, Espeseth T, Westlye LT. APOE-related biomarker profiles in nonpathological aging and early phases of Alzheimer's disease. Neurosci Biobehav Rev. (2013) 37:1322-35. doi: 10.1016/j.neubiorev.2013.05.006

84. McKenna F, Koo BB, Killiany R. Comparison of ApoE-related brain connectivity differences in early MCI and normal aging populations: an fMRI study. Brain Imaging Behav. (2016) 10:970-83. doi: $10.1007 /$ s11682-015-9451-z

85. Ye Q, Chen H, Su F, Shu H, Gong L, Xie C, et al. An inverse U-shaped curve of resting-state networks in individuals at high risk of Alzheimer's disease. J Clin Psychiatry (2018) 79:17m11583. doi: 10.4088/JCP.17m11583

86. Gong L, Shu H, He C, Ye Q, Bai F, Xie C, et al. Convergent and divergent effects of apolipoprotein E epsilon4 and epsilon2 alleles on amygdala functional networks in nondemented older adults. Neurobiol Aging (2017) 54:31-9. doi: 10.1016/j.neurobiolaging.2017.02.013

87. Thambisetty M, An Y, Kinsey A, Koka D, Saleem M, Guntert A, et al. Plasma clusterin concentration is associated with longitudinal brain atrophy in mild cognitive impairment. Neuroimage (2012) 59:212-7. doi: 10.1016/j.neuroimage.2011.07.056

88. Bai F, Shi Y, Yuan Y, Xie C, Zhang Z. Immunity factor contributes to altered brain functional networks in individuals at risk for Alzheimer's disease: neuroimaging-genetic evidence. Brain Behav Immun. (2016) 56:84-95. doi: 10.1016/j.bbi.2016.02.015

89. Bai F, Xie C, Yuan Y, Shi Y, Zhang Z. Promoter haplotypes of interleukin-10 gene linked to cortex plasticity in subjects with risk of Alzheimer's disease. Neuroimage Clin. (2018) 17:587-95. doi: 10.1016/j.nicl.2017.11.019

90. Zhuang L, Liu X, Xu X, Yue C, Shu H, Bai F, et al. Association of the interleukin 1 beta gene and brain spontaneous activity in amnestic mild cognitive impairment. J Neuroinflamm. (2012) 9:263. doi: 10.1186/1742-2094-9-263
91. Sun DM, Chen HF, Zuo QL, Su F, Bai F, Liu CF. Effect of PICALM rs3851179 polymorphism on the default mode network function in mild cognitive impairment. Behav Brain Res. (2017) 331:225-32. doi: 10.1016/j.bbr.2017.05.043

92. Seddighi S, Varma VR, An Y, Varma S, Beason-Held LL, Tanaka T, et al. SPARCL1 accelerates symptom onset in Alzheimer's disease and influences brain structure and function during aging. J Alzheimer's Dis. (2018) 61:401-14. doi: 10.3233/JAD-170557

93. Serra L, Mancini M, Cercignani M, Di Domenico C, Spano B, Giulietti $\mathrm{G}$, et al. Network-based substrate of cognitive reserve in Alzheimer's disease. J Alzheimer's Dis. (2017) 55:421-30. doi: 10.3233/JAD-1 60735

94. Bozzali M, Dowling C, Serra L, Spano B, Torso M, Marra C, et al. The impact of cognitive reserve on brain functional connectivity in Alzheimer's disease. $J$ Alzheimer's Dis. (2015) 44:243-50. doi: 10.3233/JAD-141824

95. Franzmeier N, Buerger K, Teipel S, Stern Y, Dichgans M, Ewers M. Cognitive reserve moderates the association between functional network anti-correlations and memory in MCI. Neurobiol Aging (2017) 50:152-62. doi: 10.1016/j.neurobiolaging.2016. 11.013

96. Franzmeier N, Caballero MAA, Taylor ANW, Simon-Vermot L, Buerger $\mathrm{K}$, Ertl-Wagner B, et al. Resting-state global functional connectivity as a biomarker of cognitive reserve in mild cognitive impairment. Brain Imaging Behav. (2017) 11:368-82. doi: 10.1007/s11682-016-9599-1

Conflict of Interest Statement: The authors declare that the research was conducted in the absence of any commercial or financial relationships that could be construed as a potential conflict of interest.

Copyright (c) 2018 Lin, Xing and Han. This is an open-access article distributed under the terms of the Creative Commons Attribution License (CC BY). The use, distribution or reproduction in other forums is permitted, provided the original author(s) and the copyright owner(s) are credited and that the original publication in this journal is cited, in accordance with accepted academic practice. No use, distribution or reproduction is permitted which does not comply with these terms. 